\title{
Leptomeningeal metastasis of multiple myeloma
}

\author{
Metástases leptomeníngeas de mieloma múltiplo \\ Cristiane Máximo da SILVA', Jamison Menezes de SOUZA², Roberto Queiroz dos SANTOS ${ }^{3,4}$, Mariana SPITZ1,5
}

A 65-year-old female with multiple myeloma diagnosed one year before developed tonic-clonic seizures. She had a good initial response to chemotherapy. Brain magnetic resonance imaging (MRI; Figure 1) and cerebrospinal fluid (CSF; Figure 2) analysis showed central nervous system myelomatous infiltration. Intrathecal chemotherapy and dexamethasone were prescribed and a follow-up CSF sample was negative for plasmocytes. Neurologic manifestations of multiple myeloma are not uncommon and include spinal compression and peripheral neuropathy ${ }^{1}$. Central nervous system myelomatosis, on the other hand, is rare. The workup for the diagnosis includes brain MRI and CSF analysis. CSF cytology has a sensitivity of $50-60 \%$ and a specificity over $95 \%{ }^{2}$. Prognosis is poor.

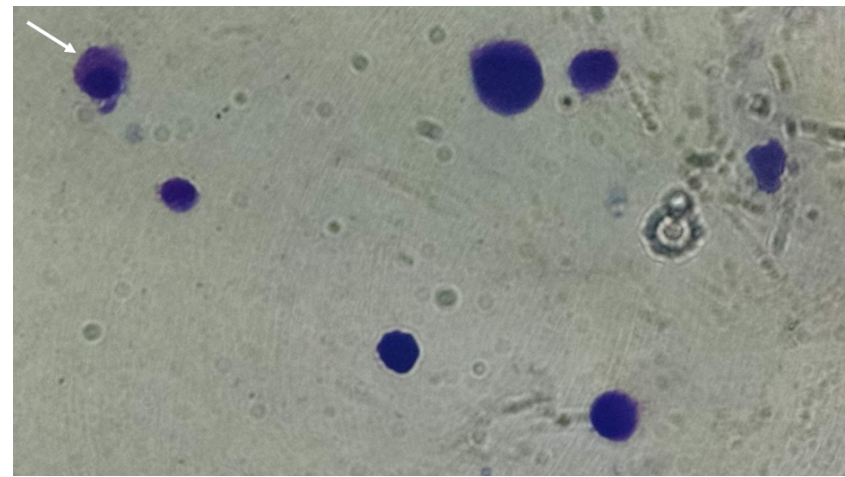

Figure 2. Cerebrospinal fluid specimen with myelomatous cells (arrow). The stain employed was May-Grunwald-Giemsa. Atypical plasmocytes found in the cerebrospinal fluid were subsequently confirmed by immunophenotyping.



Figure 1. Sagital postcontrast T1-weighted image (1A) shows mixed pachymeningeal and leptomeningeal thickening forming nodules and intense gadolinium enhancement. Axial T2-weighted image (1B) and coronal T1-weighted image (1C) show one of these nodules extending into subjacent brain parenchyma with perilesional edema.

${ }^{1}$ Hospital Federal dos Servidores do Estado, Serviço de Neurologia, Rio de Janeiro RJ, Brazil. ${ }^{2}$ Hospital Federal dos Servidores do Estado, Serviço de Hematologia, Rio de Janeiro RJ, Brazil. ${ }^{3}$ Hospital Federal dos Servidores do Estado, Serviço de Radiologia, Rio de Janeiro RJ, Brazil. ${ }^{4}$ Americas Medical City, Rio de Janeiro RJ, Brazil.

5Universidade do Estado do Rio de Janeiro, Hospital Universitário Pedro Ernesto, Serviço de Neurologia, Rio de Janeiro RJ, Brazil.

Cristiane Máximo da SILVA (iD https://orcid.org/0000-0003-4298-3140; Jamison Menezes de SOUZA (iD https://orcid.org/0000-0001-8903-7587; Roberto Queiroz dos SANTOS (iD) https://orcid.org/0000-0002-4681-8997; Mariana SPITZ (DD https://orcid.org/0000-0001-7548-2313

Correspondence: Cristiane Máximo da Silva; E-mail: cristmaximo@gmail.com

Conflict of interest: There is no conflict of interest to declare.

Authors' contributions: All authors contributed equally to the article's conception, writing, and review.

Received on April 4, 2020; Received in its final form on July 23, 2020; Accepted on July 25, 2020. 


\section{References}

1. Brum M, António AS, Guerreiro R. Myelomatous meningitis: a rare neurological involvement in complete remission of multiple myeloma.J Neurol Sci. 2014 May;340(1-2):241-2. https://doi. org/10.1016/j.jns.2014.03.015
Bommer M, Kull M, Teleanu V, Schwarzwälder P, Feuring-Buske M, Kroenke J, et al. Leptomeningeal myelomatosis: A rare but devastating manifestation of multiple myeloma diagnosed using cytology, flow cytometry, and fluorescent in situ hybridization. Acta Haematol. 2018 Aug;139(4):247-54. https://doi.org/10.1159/000489484 